\title{
High-Color-Purity Subtractive Color Filters with a Wide Viewing Angle Based on Plasmonic Perfect Absorbers
}

Kyu-Tae Lee, Sungyong Seo, and L. Jay Guo*

It has long been believed that color filters received a substantial amount of attention as a vital element for diverse applications, such as image sensors, liquid crystal displays, light emitting diodes, and optical measurement systems. Traditional color filters, however, make use of colorant organic dyes that are highly sensitive to long-term heat exposure, strong intensity of ultraviolet irradiation, and moisture, which results in performance deterioration over time. ${ }^{[1]}$ In recent years, a great effort has been made to develop structural color filters that provide distinct advantages over existing color filters. Various schemes have been suggested based on plasmonic nanoresonators, ${ }^{[2-4]}$ perforated metal films, ${ }^{[5-7]}$ metal-dielectric multilayer systems, ${ }^{[8]}$ photonic crystals, ${ }^{\left[{ }^{[-13]}\right.}$ nanovolcano arrays, ${ }^{[14]}$ nanorod arrays, ${ }^{[15]}$ and nanowires. ${ }^{[16]}$ Even though these approaches show a performance enhancement in terms of the efficiency, durability, and dimension, there still have been great difficulties in achieving an angle insensitive behavior that is of critical importance in a wide variety of fields. In addition, a spectral response of the structural color filters based on the above concepts has a relatively broad profile whose full width at half maximum (FWHM) is in general broader than $100 \mathrm{~nm}$ and also encompasses a large amount of off-resonance wavelength components, thereby greatly reducing the purity of the color. These drawbacks significantly limit the practical applications and therefore it is indeed necessary to develop a platform that offers not only incident angle independent performance but also narrow FWHM with highly suppressed off-resonance wavelengths.

In the present study, we propose and experimentally demonstrate subtractive structural color filters with enhanced color purity employing a nearly perfect plasmonic absorption behavior in the metal-insulator-metal (MIM) configuration for addressing aforementioned challenges simultaneously. The proposed devices exhibit almost perfect absorption property $(\approx 97 \%)$ at a resonance wavelength and thus its complementary spectrum is reflected, being able to produce reflective colors. Such sharp resonance or color characteristic is retained over a large angle of incidence up to $\pm 80^{\circ}$ without sacrificing the intensity and bandwidth. The FWHM of the fabricated samples is only $36 \mathrm{~nm}$ with highly suppressed off-resonance wavelengths, thus accomplishing improved purity of the color.

A device structure under investigation, as shown in Figure 1a, is composed of the MIM resonator on a silicon substrate. We select silver $(\mathrm{Ag})$ for a metallic layer since it has lower

K.-T. Lee, S. Seo, Prof. L. J. Guo

Department of Electrical Engineering and

Computer Science

The University of Michigan

Ann Arbor, MI 48109, USA

E-mail:guo@umich.edu

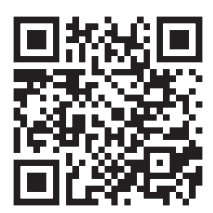

DOI: 10.1002/adom.201400533 optical absorption loss at visible frequencies. The dispersion curve of the MIM structure consisting of two semi-infinite Ag metallic mirrors separated by $\lambda_{\mathrm{sp}} / 4$-thick air medium is exhibited in Figure 1b. The wave vector and frequency are normalized with respect to $k_{\mathrm{p}}\left(=\omega_{\mathrm{p}} / c\right)$ and $\omega_{\mathrm{p}}$, where $\omega_{\mathrm{p}}$ is the plasma frequency and $c$ is the speed of light in free space. As is seen from the figure, there are three discrete modes: I. Propagating surface plasmon polariton (SPP) mode, II. Quasi-bound mode, III. Radiative mode. The SPP and radiative modes correspond to the antisymmetric solution and quasi-bound mode to the symmetric one in the MIM structure. The SPP mode has real $k_{z}$ and imaginary $k_{x}$, thus propagating along the metal-insulator interface (i.e., $z$-direction) while the quasi-bound mode has real $k_{x}$ and imaginary $k_{z}$, thus radiating into the forward direction (i.e., $x$-direction). The radiative mode has real $k_{z}$ and real $k_{x}$. The analytical dispersion characteristics of these modes are derived in ref. ${ }^{[17]}$ and more detailed investigations can be given in refs. ${ }^{[18-20]}$. Here, we will mainly focus on the mode II that can be easily coupled without any external mechanism since its dispersion curve lies above the air light line. When the parallel component of the wave vector $\left(k_{\mathrm{II}}\right)$ goes to the infinity, the mode II's dispersion frequency $\left(\omega_{\text {II }}\left(k_{\text {II }}\right)\right)$ moves toward the SPP frequency in free space $\left(\omega_{\mathrm{sp}}=\frac{\omega_{p}}{\sqrt{2}}\right)$ occurring at the interface between air and metal. From the SPP dispersion expression $\left(k_{\mathrm{II}}=\frac{\omega}{c}\left(\frac{\varepsilon_{\text {metal }} \varepsilon_{\text {insulator }}}{\varepsilon_{\text {metal }}+\varepsilon_{\text {insulator }}}\right)^{1 / 2}\right)$, it is obvious that the permittivity of the metallic layer should be the same to the permittivity of the insulator with opposite sign at the SPP frequency (i.e., $\varepsilon_{\text {metal }}=-\varepsilon_{\text {insulator }}$ ). When the thickness ( $\left.t\right)$ of the insulating layer is equal to $\frac{\pi c}{2 \omega_{\mathrm{sp}} \sqrt{\varepsilon_{\text {insulator }}}}$, the dispersion frequency at $k_{\mathrm{II}}=0$ $\left(\omega_{I I}\left(k_{\mathrm{II}}=0\right)\right)$ will correspond to the SPP frequency. Simultaneously satisfying above two conditions enables the high angular tolerant resonance to appear at the SPP frequency, thus showing a flat dispersion band in the MIM configuration. ${ }^{[17,21]}$ Such flat dispersion characteristics at visible frequency will be exploited in order to create reflective colors with wide viewing angle.

Real parts of the permittivity of Ag, titanium dioxide $\left(\mathrm{TiO}_{2}\right)$, and amorphous silicon (a-Si) materials as a function of wavelength measured by a spectroscopic ellipsometer (M-2000, J. A. Woollam) are depicted in Figure 2. For easy comparison, the permittivity of Ag with opposite sign is plotted by a dotted line. As shown in the inset of the figure, absolute values of the permittivity of Ag are found to be 5.302 and 17.580 that demonstrate excellent match with the permittivity of $\mathrm{TiO}_{2}$ (5.325) and a-Si (17.585) at $420 \mathrm{~nm}$ and $627 \mathrm{~nm}$, respectively. It is thus expected that the resonance behaviors with a low sensitivity to the angle of incidence appear with the proper thickness of the 
(a)

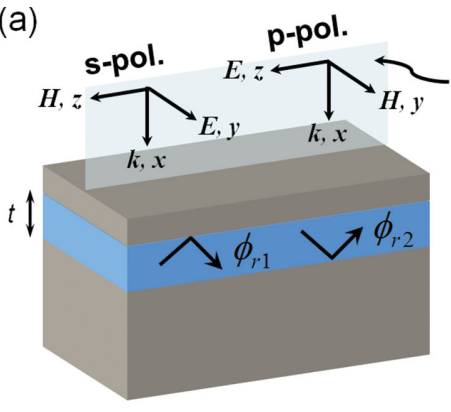

(b)

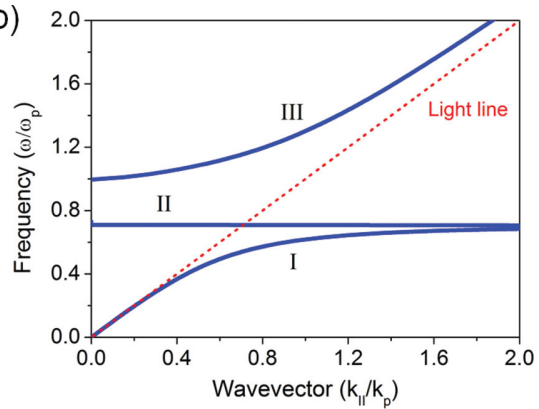

Figure 1. a) A schematic representation of the proposed angle insensitive color filters exploiting a perfect absorption property in a microcavity, composed of metal-insulator-metal (MIM). For producing the reflective yellow $(420 \mathrm{~nm})$ and cyan $(627 \mathrm{~nm})$ colors, $\mathrm{TiO}_{2}$ and a-Si are used as insulator layers. $\mathrm{Ag}$ is chosen as a metal mirror due to its lower absorption at visible frequency. b) Dispersion curve of a MIM structure comprising two Ag mirrors separated by an air with the thickness of $\lambda_{\mathrm{sp}} / 4$.

middle insulating layer (either $\mathrm{TiO}_{2}$ or a-Si). An adequate thickness of the insulator is calculated exhibiting that $46 \mathrm{~nm}$ of $\mathrm{TiO}_{2}$ and $35 \mathrm{~nm}$ of a-Si layers are needed in order to create the resonance at the SPP frequency.

In Figure 3, the calculated total round-trip phase shift, encompassing the reflection and propagation phase shifts, exhibited at the SPP frequency as a function of the incidence

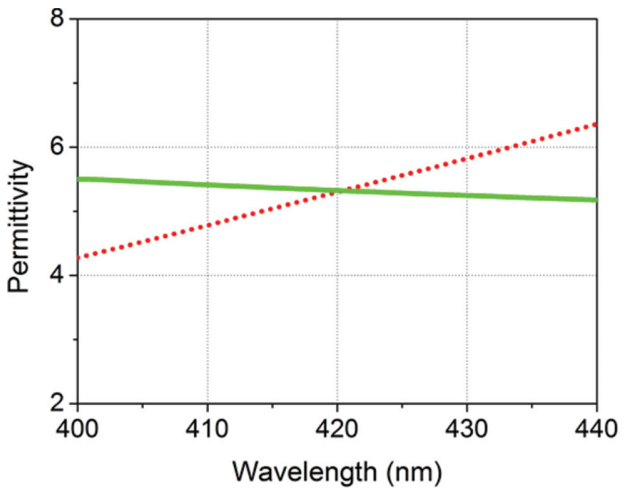

angle. The reflection phase shift is computed from the reflection coefficient for p-polarization, that is, $r=\frac{r_{12}+r_{23} e^{2 j k}}{1+r_{12} r_{23} e^{2 j k}}$ where $\quad r_{p q}=\frac{n_{p} \cos \left(\theta_{q}\right)-n_{q} \cos \left(\theta_{p}\right)}{n_{p} \cos \left(\theta_{q}\right)+n_{q} \cos \left(\theta_{p}\right)}$ and $k=\left(\frac{2 \pi}{\lambda}\right) n_{2} t_{2} \cos \left(\theta_{2}\right)$. Medium 1, 2, and 3 correspond to the top thin $\mathrm{Ag}$, insulator,

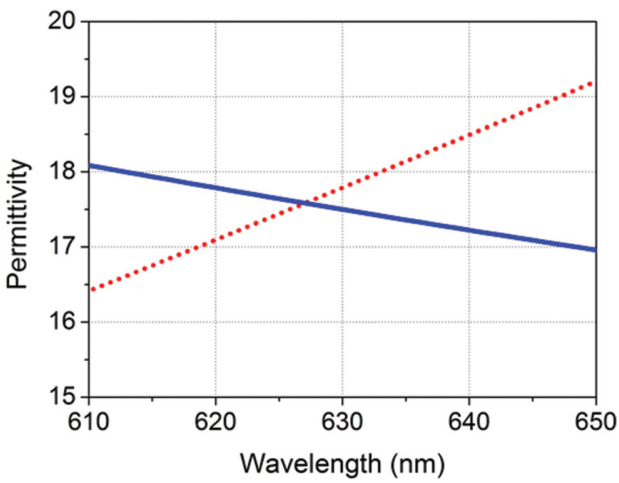

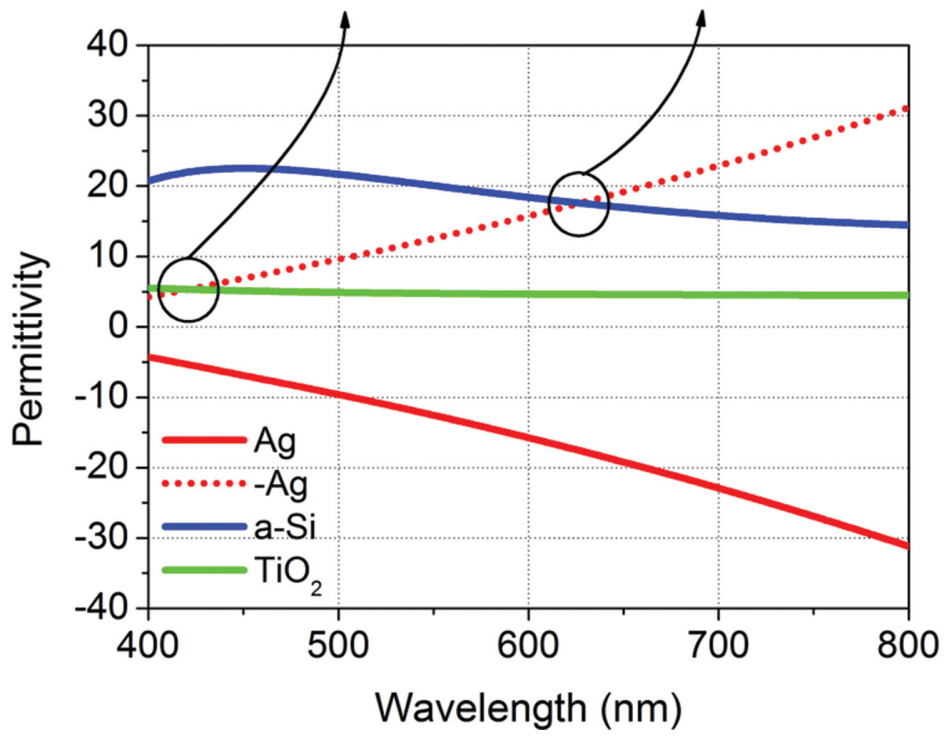

Figure 2. Real parts of the dielectric constant of $\mathrm{Ag}, \mathrm{TiO}_{2}$, and a-Si as a function of wavelength measured by a spectroscopic ellipsometer (M-2000, J. A. Woollam). The inset shows an enlarged view of the dielectric constant in the vicinity of each surface plasmon resonance wavelength. 
(a)

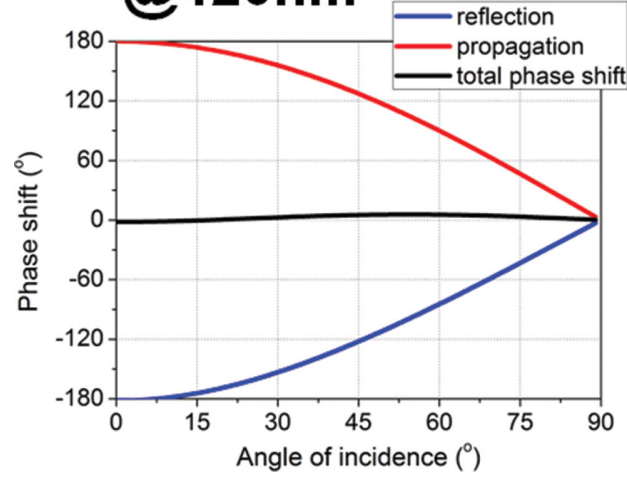

(b)

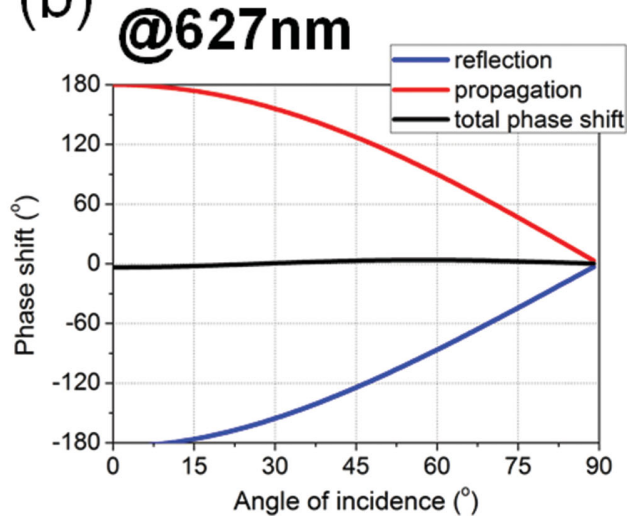

Figure 3. The net phase shift (black solid line), including the reflection phase shift (blue solid line) and the propagation phase shift (red solid line) obtained from the round-trip in the MIM resonator as a function of the angle of incidence at a) $420 \mathrm{~nm}$ and b) $627 \mathrm{~nm}$ that correspond to the surface plasmon resonance wavelength of each resonator.

and bottom thick Ag layer, respectively. And the propagation phase shift through the insulator medium is calculated by $\left(\frac{2 \pi}{\lambda}\right) \sqrt{\varepsilon_{\text {insulator }}} 2 t_{\text {insulator }} \cos \theta$. When satisfying the second condition, the accumulation of the phase shift during the light propagation through the insulating layer approximately cancels out the phase shift obtained from the reflection at the interface between metal and insulator. From the calculation, the sum of the two reflection phase shifts $\left(\phi_{r 1}\right.$ and $\left.\phi_{r 2}\right)$ increases with increasing the angle of incidence (i.e., from $-180^{\circ}$ to $0^{\circ}$ ), whereas the phase shift associated with the round-trip propagation decreases with increasing the incidence angle (i.e., $180^{\circ}$ to $0^{\circ}$ ), showing that the sum of the reflection and propagation phase shifts leads to the zero (i.e., $2 m \pi$ ( $m$ is an integer)) for all angles of incidence. This signifies that the resonance stays at the SPP wavelength over a large angular range under the appropriate condition.

Next, we investigate an optical absorption characteristic in the MIM resonator system near the condition of a critical coupling. When the internal loss rate $\left(\gamma_{\text {int }}\right)$ is equal to the radiation coupling rate $\left(\gamma_{\mathrm{rad}}\right)$, the $100 \%$ optical absorption can be achieved that is attributed to the perfect destructive interference between the incident light wave and the reflected light wave yielding the total absorption at the critical condition. ${ }^{[22-25]}$ Figure $4 \mathrm{a}, \mathrm{b}$ show simulated contour plots of the absorption as a function of wavelength and the thickness of a top Ag film. Nearly perfect absorptions are attained with $48.7 \mathrm{~nm}$ and $22.8 \mathrm{~nm}$ of the top Ag layer for the yellow $(420 \mathrm{~nm})$ and cyan $(627 \mathrm{~nm})$, respectively. In Figure $4 c, d$, simulated absorption spectra for three different cases of overcoupling (dark yellow solid line, $\gamma_{\text {int }}<\gamma_{\text {rad }}$ ), critical coupling (red solid line, $\gamma_{\text {int }}=\gamma_{\mathrm{rad}}$ ), and undercoupling (blue solid line, $\gamma_{\text {int }}>\gamma_{\text {rad}}$ ) are illustrated. As can be seen from the figure, a nearly total absorption performance is obtained at the critical coupling condition, while the absorption efficiency starts decreasing in the over and undercoupling regimes. Also, as the thickness of the top Ag layer increases the resonance is slightly shifted toward the shorter wavelength range due to the varied reflection phase shift at the top metal-insulator interface depending on the metal thickness. The resonance also gets sharper with increasing the thickness of the metal, which is attributed to the enhanced reflectivity of the metal surface (i.e., high Q-factor). Normalized electric field profiles at the resonance wavelength are shown in Figure 4e,f exhibiting a symmetric distribution of the field in the MIM resonator.

Figure 5a describes simulation profiles of the reflectance (solid lines) and the absorption (dotted lines) of the designed structure. As there is no transmittance due to an optically thick bottom metallic substrate, the complementary spectrum of the absorption is the reflectance according to the well-known relation $(1=R+A)$. It is noteworthy to mention that the top metal has to be somewhat thin that allows incident light wave to traverse an entire structure and simultaneously provides a sufficient reflection phase change to achieve the wide angle plasmonic resonance effect. From the study in a previous section, we found that the top Ag layer for the yellow color (420 nm) needs to be $48.7 \mathrm{~nm}$ that is too thick for incident light to pass through the whole resonator system. Although we lose the absorption efficiency to some extent, the thickness of the top Ag layer for creating the yellow color $(420 \mathrm{~nm})$ is designed to be $33 \mathrm{~nm}$ (i.e., overcoupling regime). The thickness of the top Ag layer for the cyan $(627 \mathrm{~nm})$ color is $23 \mathrm{~nm}$ that enables the resonator to operate at the critical coupling. Varying the insulator material yields the change in the SPP frequency and as a result the reflective color can be tuned. We note that the magenta color can also be made by using zinc telluride ( $\mathrm{ZnTe}$ ) whose permittivity is fairly similar to the permittivity of $\mathrm{Ag}$ at $\approx 525 \mathrm{~nm}$. The deposition of $\mathrm{TiO}_{2}$ and $\mathrm{Ag}$ was done by the electron-beam evaporation and a-Si was deposited by using plasmaenhanced chemical vapor deposition (PECVD). The experimental spectra of the reflectance are measured by using a film thickness measurement instrument (F20, Filmetrics) integrated with a spectrometer and white light source unit, and the optical absorption profiles are attained from $1-R$ relation as shown in Figure $5 b$ that shows fair agreement with the calculated profiles. A non-smooth surface of each layer results in the scattering light loss, making the bandwidth of the measured data slightly wider. It is clear that the discrepancy between calculated and measured spectra is large for the yellow (420 nm), implying that the scattering loss of the yellow-colored device $(420 \mathrm{~nm})$ is more significant than that of the cyan sample $(627 \mathrm{~nm})$. This 
(a)

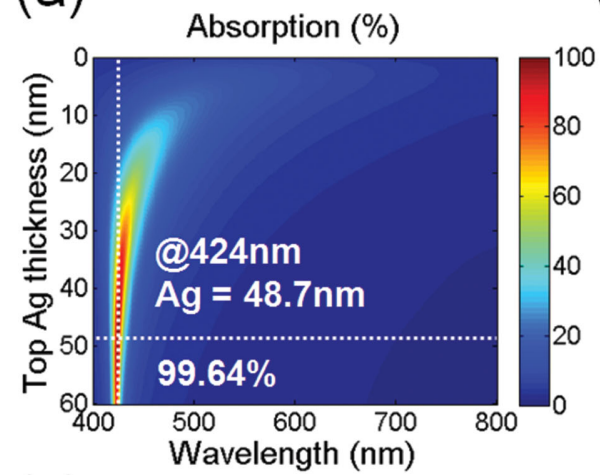

(c)

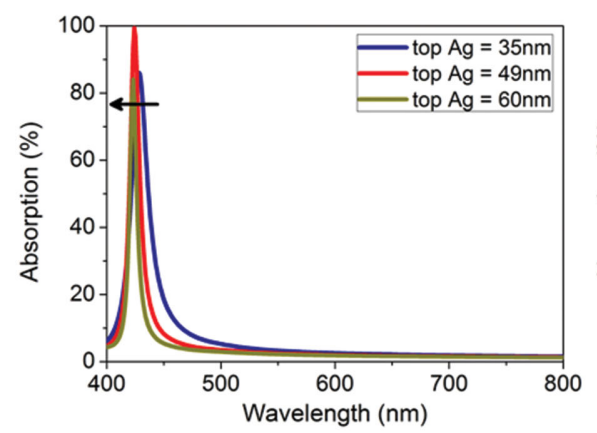

(e) @424nm

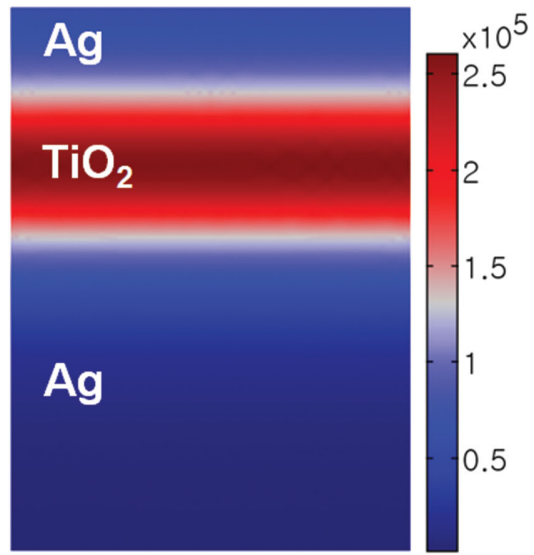

(b)

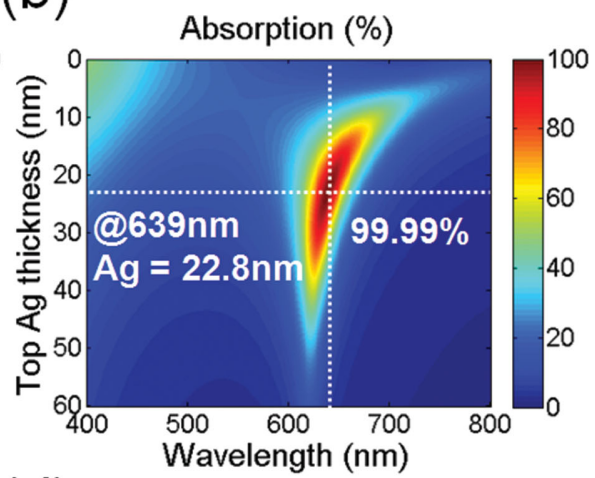

(d)

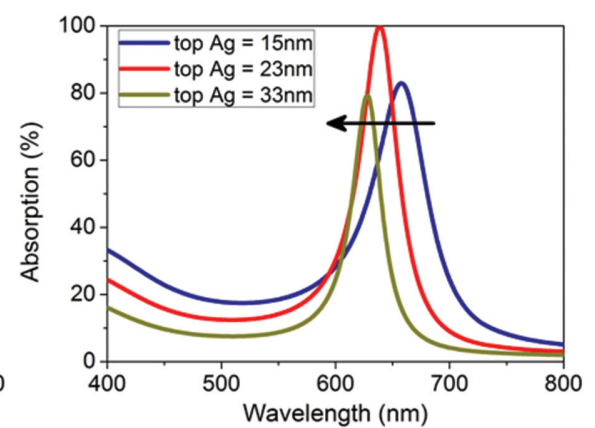

(f) @639nm

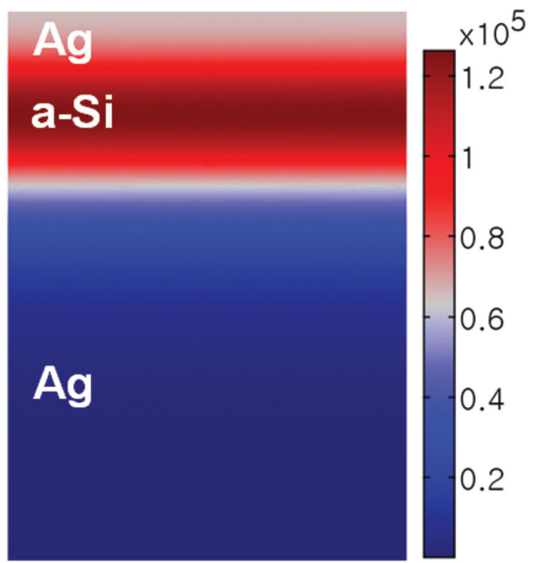

Min: 286.318

Figure 4. The calculated contour plots of the absorption for a) yellow $(420 \mathrm{~nm})$ and b) cyan $(627 \mathrm{~nm})$ as a function of wavelength and the thickness of top $\mathrm{Ag}$ layer. Almost perfect absorption is observed under the critical coupling condition for both cases. The calculated absorption spectra corresponding to the under (blue), critical (red), and over (dark yellow) coupling conditions for c) yellow (420 nm) and d) cyan (627 nm). The electric field profiles at e) $424 \mathrm{~nm}$ and f) $639 \mathrm{~nm}$ showing a highly concentrated field in the insulator layer with symmetric distribution in the MIM resonator under the critical coupling condition.

is in accordance with the Rayleigh scattering cross-section $\left(\sigma_{s}=\frac{2 \pi^{5}}{3} \frac{d^{6}}{\lambda^{4}}\left(\frac{n^{2}-1}{n^{2}+2}\right)^{2}\right)$ that is inversely proportional to fourth power of wavelength. $d$ is the size of the particle and the $n$ represents the index of refraction. We note that such undesired scattering loss can be reduced by depositing perylenetetracarboxylic bis-benzimidazole (PTCBI) before the thin Ag film deposition or ultra-smooth Al-Ag alloying film. ${ }^{[26-28]}$ It is also important to note that the FWHM of the reflectance is only $36 \mathrm{~nm}$ with highly suppressed off-resonance wavelength components from experimental results, thereby yielding improved color purity.

Finally, we study the angular behavior of the proposed color filters. As described earlier, we utilize the mode that has the flat dispersion characteristic in the MIM configuration arising from the fact that the phase change acquired by the light propagating through the cavity approximately compensates the reflection 
(a) Simulation

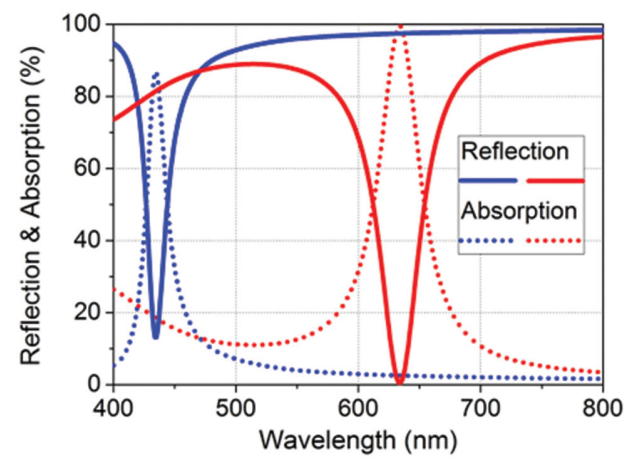

(b) Experiment

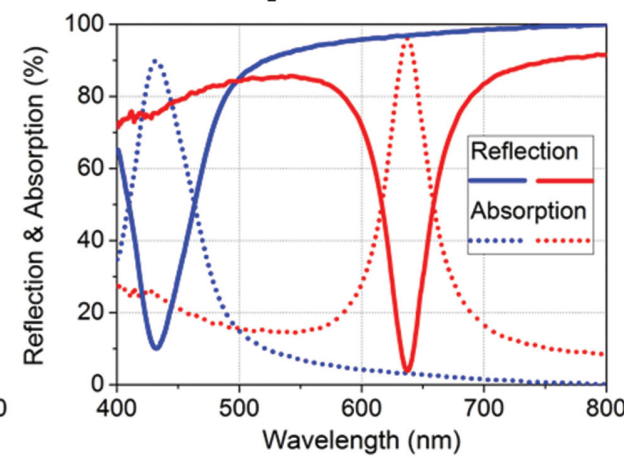

(Top Ag film thickness)

Yellow $(420 \mathrm{~nm}): 33 \mathrm{~nm} \&$ Cyan $(630 \mathrm{~nm}): 23 \mathrm{~nm}$

Figure 5. a) The calculated reflection (solid lines) and absorption (dotted lines) spectra of the proposed color filters at normal incidence. The thicknesses of the $\mathrm{TiO}_{2}$ and a-Si layers necessary for creating the yellow $(420 \mathrm{~nm})$ and cyan $(627 \mathrm{~nm})$ reflective colors are $46 \mathrm{~nm}$ and $35 \mathrm{~nm}$, respectively. The thicknesses of the top Ag film for creating yellow $(420 \mathrm{~nm})$ and cyan $(627 \mathrm{~nm})$ are 33 and $23 \mathrm{~nm}$, respectively. b) The measured spectra corresponding to the profiles in a) exhibiting slightly broader profiles due to the scattering loss induced by the non-smooth surfaces.

phase changes, suggesting that our devices show the directional independent performance. Such phase compensation related to non-trivial reflection phase shifts obtained at the metalsemiconductor interface to form a resonance in an ultra-thin semiconductor medium as compared to the wavelength of incident light was recently investigated. ${ }^{[29,30]}$ To validate such angle insensitive performance of our designs, the transfer matrix method-based simulation is carried out. As expected, the simulated dispersion curves exhibit that the resonance remains at the surface plasmon resonance wavelength over a large incident angular range for p-polarization as exhibited in Figure 6a,b. From the simulation, the FWHMs of the yellow $(420 \mathrm{~nm})$ and cyan $(627 \mathrm{~nm})$ devices are $20 \mathrm{~nm}$ and $31 \mathrm{~nm}$, respectively, and its high efficient properties are retained up to $\pm 80^{\circ}$ from the simulation. The dispersion properties of the fabricated devices are measured by the spectroscopic ellipsometer (M-2000, J. A. Woollam) from $45^{\circ}$ to $80^{\circ}$ and illustrated in Figure $6 \mathrm{c}, \mathrm{d}$, which agree well with the calculated results. The measured data show $51 \mathrm{~nm}$ and $36 \mathrm{~nm}$ of the FWHM for the yellow $(420 \mathrm{~nm})$ and cyan $(627 \mathrm{~nm})$ filters, displaying relatively broader FWHM than the simulated profile that is because of the light scattering loss. It is obvious that the measured spectra have low background noise level that primarily determines the purity of the color. It should be noted that the resulting spectrum of the (a)

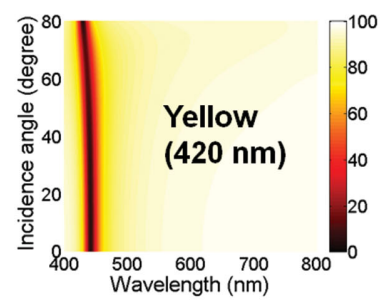

(c) Experiment

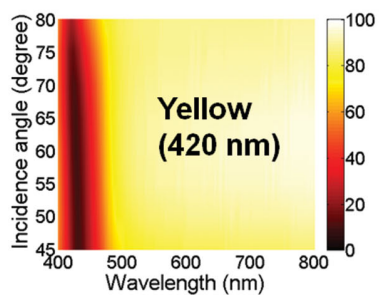

(b)

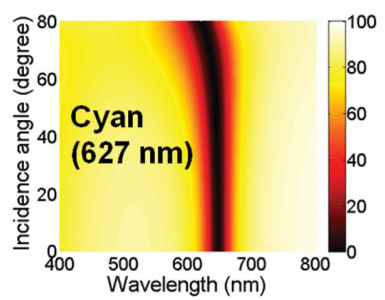

(d)

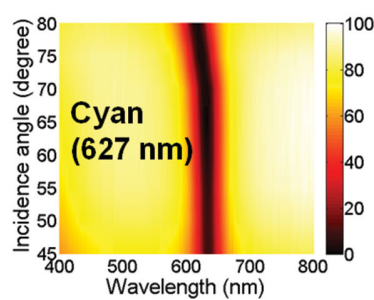

(e)

Angle: $\mathbf{2 0}^{\circ}$

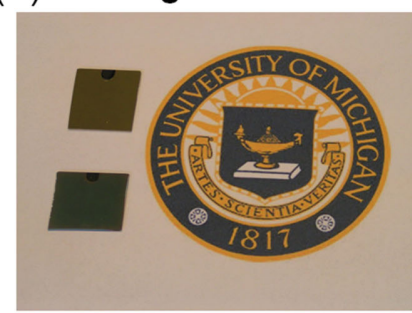

Angle: $70^{\circ}$

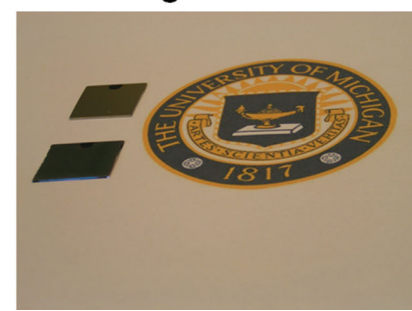

Figure 6. The calculated dispersion curves of a) yellow (420 nm) and b) cyan (627 nm) show that the surface plasmon resonance wavelengths stay approximately constant over a wide incident angular range, thus validating the angle insensitive characteristic in the MIM resonator. c, d) The measured dispersion curves corresponding to the those in $(\mathrm{a}, \mathrm{b})$. The angle resolved reflection spectra are measured by a spectroscopic ellipsometer (M-2000, J. A. Woollam) at the incidence angle from $45^{\circ}$ to $80^{\circ}$ for p-polarization. e) The optical images of the fabricated devices illuminated by the indoor light at two different oblique angles of incidence displaying no color change. 
fabricated samples keeps its intensity and the bandwidth over a wide incident angular range. The photographs of the fabricated samples at oblique angles of incidence are shown in Figure 6e. The original reflective colors remain the same even at a large angle, therefore confirming the angle robust performance of the proposed designs. The color creation with wide viewing angle is highly desired in a variety of areas, such as color displays and light emitting diodes.

To sum up, we have demonstrated the subtractive color filters capable of producing the reflective colors with narrow bandwidth based on the plasmonic perfect absorption characteristic over a broad incident angular range in the MIM structure. There is no change in the reflected color with improved purity of the color even at a very large angle of incidence, $\pm 80^{\circ}$. Presented strategy can pave the way for many applications such as color display devices, optical measurements, light modulators, and image sensors.

\section{Experimental Section}

Device Fabrication: A silicon substrate was piranha cleaned (mixture of $\mathrm{H}_{2} \mathrm{SO}_{4}$ and $\mathrm{H}_{2} \mathrm{O}_{2}$ with 1:1 volume ratio) for $25 \mathrm{~min}$. $\mathrm{Ag}$ and $\mathrm{TiO}_{2}$ were deposited by the e-beam evaporation at a rate of $5 \AA \mathrm{s}^{-1}$ with a high vacuum $\left(1 \times 10^{-6} \mathrm{mbar}\right)$ and a-Si was deposited by using the PECVD at a rate of $1 \AA \mathrm{s}^{-1}\left(\mathrm{SiH}_{4}\right.$ gas with $13.56 \mathrm{MHz} \mathrm{RF}$ power).

Simulation and Measurement: We carried out the simulation using a transfer matrix method based on the refractive indices measured by a spectroscopic ellipsometer (M-2000, J. A. Woollam). We performed the spectral reflectance measurements of the fabricated colored devices at normal incidence by using a thin-film measurement instrument (F20, Filmetrics) integrated with a white light source and spectrometer. The angle resolved reflection spectra measurement for p-polarization were performed by using the spectroscopic ellipsometer (M-2000, J. A. Woollam).

\section{Acknowledgements}

The authors would like to thank B. S. Song for $\mathrm{TiO}_{2}$ and $\mathrm{Ag}$ deposition. The authors would also like to acknowledge the support by the NSF (ECCS 1202046 and DMR 1120923). K.T.L. and S.S. contributed equally to this work.
[1] R. W. Sabnis, Displays 1999, 20, 119.

[2] T. Xu, Y.-K. Wu, X. Luo, L. J. Guo, Nat. Commun. 2010, 1, 59.

[3] Y. S. Do, J. H. Park, B. Y. Hwang, S.-M. Lee, B.-K. Ju, K. C. Choi, Adv. Opt. Mater. 2013, 1, 133.

[4] A. S. Roberts, A. Pors, O. Albrektsen, S. I. Bozhevolnyi, Nano Lett. 2014, 14, 783.

[5] H.-S. Lee, Y.-T. Yoon, S.-S. Lee, S.-H. Kim, K.-D. Lee, Opt. Express 2007, 15, 15457.

[6] D. Inoue, A. Miura, T. Nomura, H. Fujikawa, K. Sato, N. Ikeda, D. Tsuya, Y. Sugimoto, Y. Koide, Appl. Phys. Lett. 2011, 98, 093113.

[7] S. Yokogawa, S. P. Burgos, H. A. Atwater, Nano Lett. 2012, 12, 4349.

[8] D. Chung, C. Shin, B. Song, M. Jung, Y. Yun, S. H. Nam, C. Noh, J. Kim, S. Lee, Appl. Phys. Lett. 2012, 101, 221120.

[9] Y.-T. Yoon, H.-S. Lee, S.-S. Lee, S. H. Kim, J.-D. Park, K.-D. Lee, Opt. Express 2008, 16, 2374

[10] E.-H. Cho, H.-S. Kim, J.-S. Sohn, C.-Y. Moon, N.-C. Park, Y.-P. Park, Opt. Express 2010, 18, 27712.

[11] H. Kim, J. Ge, J. Kim, S.-E. Choi, H. Lee, H. Lee, W. Park, Y. Yin, S. Kwon, Nat. Photonics 2009, 3, 534.

[12] A. F. Kaplan, T. Xu, L. J. Guo, Appl. Phys. Lett. 2011, 99, 143111.

[13] C.-H. Park, Y.-T. Yoon, V. R. Shrestha, C.-S. Park, S.-S. Lee, E.-S. Kim. Opt. Express 2013, 21, 28783.

[14] B. Ai, Y. Yu, H. Möhwald, G. Zhang, Adv. Opt. Mater. 2013, 1, 724.

[15] G. Si, Y. Zhao, J. Lv, M. Lu, F. Wang, H. Liu, N. Xiang, T. J. Huang, A. J. Danner, J. Teng, Y. J. Liu, Nanoscale 2013, 5, 6243.

[16] K. Seo, M. Wober, P. Steinvurzel, E. Schonbrun, Y. Dan, T. Ellenbogen, K. B. Crozier, Nano Lett. 2011, 11, 1851.

[17] E. N. Economou, Phys. Rev. 1969, 182, 539.

[18] J. Chen, G. A. Smolyakov, S. R. J. Brueck, K. J. Malloy, Opt. Express 2008, 16, 14902

[19] J. A. Dionne, L. A. Sweatlock, H. A. Atwater, A. Polman, Phys. Rev. B 2005, 72, 075405.

[20] R. Zia, M. D. Selker, M. L. Brongersma, Phys. Rev. B 2005, 71, 165431.

[21] H. Shin, M. F. Yanik, S. Fan, R. Zia, M. L. Brongersma, Appl. Phys. Lett. 2004, 84, 4421.

[22] A. Yariv, Electron. Lett. 2000, 36, 321.

[23] E. Almpanis, N. Papanikolaou, J. Appl. Phys. 2013, 114, 083106.

[24] T. V. Teperik, F. J. Garcia De Abajo, A. G. Borisov, M. Abdelsalam, P. N. Bartlett, Y. Sugawara, J. J. Baumberg, Nat. Photonics 2008, 2, 299.

[25] J. Yoon, K. H. Seol, S. H. Song, R. Magnusson, Opt. Express 2010, $18,25702$.

[26] K.-T. Lee, J. Y. Lee, S. Seo, L. J. Guo, Light Sci. Appl. 2014, 3, e215.

[27] J. Y. Lee, K.-T. Lee, S. Seo, L. J. Guo, Sci. Rep. 2014, 4, 4192.

[28] C. Zhang, D. Zhao, D. Gu, H. Kim, T. Ling, Y.-K. R. Wu, L. J. Guo, Adv. Mater. 2014, 26, 5696.

Received: November 7, 2014

Revised: November 30, 2014

Published online: January 2, 2015
[29] K.-T. Lee, S. Seo, J. Y. Lee, L. J. Guo, Appl. Phys. Lett. 2014, 104 231112.

[30] K.-T. Lee, S. Seo, J. Y. Lee, L. J. Guo, Adv. Mater. 2014, 26, 6324. 